\title{
Research on the application of molecular simulation technology in enhanced oil-gas recovery engineering
}

\author{
Jianping $\mathrm{Xu}^{1,2}$, Yuanda Yuan ${ }^{1,2, *}$, Qing Xie ${ }^{1,2}$, Xuegang Wei ${ }^{1,2}$ \\ ${ }^{1}$ College of Petroleum Engineering, Xi'an Shiyou University. Xi'an 710065, China ${ }^{1}$, \\ ${ }^{2} \mathrm{MOE}$ Engineering Research Center of Development \& Management of Western Low \& Ultra-Low Permeability Oilfield, Xi'an 710065, \\ China $^{2}$
}

\begin{abstract}
In recent years, molecular simulations have received extensive attention in the study of reservoir fluid and rock properties, interactions, and related phenomena at the atomistic scale. For example, in molecular dynamics simulation, interesting properties are taken out of the time evolution analysis of atomic positions and velocities by numerical solution of Newtonian equations for all atomic motion in the system. These technologies assists conducting "computer experiments" that might instead of be impossible, very costly, or even extremely perilous to carry out. Whether it is from the primary oil recovery to the tertiary oil recovery or from laboratory experiment to field test, it is difficult to clarify the oil displacement flow mechanism of underground reservoirs. Computer molecular simulation reveals the seepage mechanism of a certain oil displacement at the microscopic scale, and enriches the specific oil displacement flow theory system. And the molecular design and effect prediction of a certain oil-displacing agent were studied, and its role in the reservoir was simulated, and the most suitable oil-displacing agent and the best molecular structure of the most suitable oil-displacing agent were obtained. To give a theoretical basic for the development of oilfield flooding technology and enhanced oil/gas recovery. This paper presents an overview of molecular simulation techniques and its applications to explore enhanced oil/gas recovery engineering research, which will provide useful instructions for characterizing the reservoir fluid and rock and their behaviors in various oil-gas reserves, and it greatly contribute to perform optimal operation and better design of production plants.
\end{abstract}

\section{Introduction}

Molecular simulation technology uses computers to simulate the structure and behavior of molecules with atomic-level molecular models ${ }^{[1,2-4,5]}$. It can give the physical quantity which can not be measured by the existing experiment of the system under study ${ }^{[6]}$, This method is an advanced method that has attracted much attention in recent years in the study of fluid and rock properties and their associated phenomena. It is one of the advanced deterministic decision making approaches for studying complicated systems at the atomistic and molecular level. It is based on statistical mechanics and quantum mechanics. After the motion of all the atoms in the system, the coordinates and motion of the atoms are analyzed by statistical average, and the properties of equilibrium adsorption, dynamics and thermodynamics can be extracted $\mathrm{d}^{[7,8,9]}$. In this way, so-called "computer experiments" can be conducted to investigate the behavior of systems and physical and chemical phenomena $^{[10-11]}$. As shown in Figure. 1, Due to the emergence of this technology, there are not only one more research method to explore the mechanism, but also more tools to support the experiment and theory.

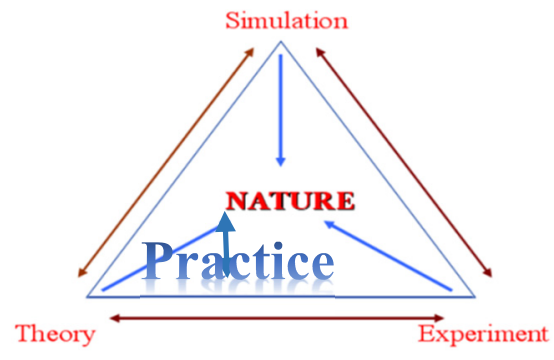

Figure 1. Relationship between simulation, theory and experiment.

Molecular simulation is used to study various equilibrium and transmission characteristics of reservoir fluid, simulate different fluid characteristics and fluid related phenomena, and make researchers see the three-dimensional visualization of molecular motion behavior $^{[12]}$.Many researches have begun to take in higher levels of rock heterogeneity (pore size, mineral composition, and more complex multicomponent reservoir fluids) into molecular simulations ${ }^{[13,14-16]}$. Such advances of molecular simulation techniques are valuable for characterization and analysis of oil and gas reservoirs because they provide a understanding of their production mechanisms and flow-related phenomena at a

\footnotetext{
*Correspondence should be addressed to Yuanda Yuan; 792343379@qq.com*
} 
level that is difficult to study experimentally.

In this paper, we first summarize the theory of molecular simulation. Then the latest application of this modeling method in reservoir fluid and rock characterization is reviewed. The practical challenges and new research directions of molecular simulation are also discussed. Molecular simulation technology has exhibited great potential in elucidating the atomic and molecular properties of complicated phenomena in reservoir researches, and it is an excellent characterization technique in view of the inherent complexities and restrictions of oilfield developments. The purpose of this review is to highlight the wide application of molecular simulation and the many opportunities it offers to help scholars gain the understanding of complex physico-chemical phenomena of interest in reservoir analysis. Currently, there is a general lack of understanding of the workflow and standards for EOR engineering related modeling using molecular simulations, the purpose of this article is to further identify and emphasize the necessity of developing such a workflow that will ultimately make this powerful and beneficial simulation technology easier to use in the oil-gas industry.

\section{Overview of molecula simulation}

With the development of computer applications in scientific research, molecular simulation technique is a combination of computer science and basic science. It relies on the software simulation platform and uses the theory method to simulate the motion behavior of molecules in the micro world. It is a low-cost innovation technology, which can intuitively represent the molecular and micro scale under the reservoir condition ${ }^{[17,18,19]}$. Implementation: Firstly, the molecular model was built on the computer software, including the detailed description of the atomic position of the research object and the establishment of the intermolecular interaction force field. Then, the position and motion of the molecules were statistically averaged with appropriate statistical mechanical relations and algorithms in order to calculate the required macroscopic properties ${ }^{[1,20]}$. The main methods of molecular simulations are as follows, Figure 2. shows the corresponding methods in different time and space scales.

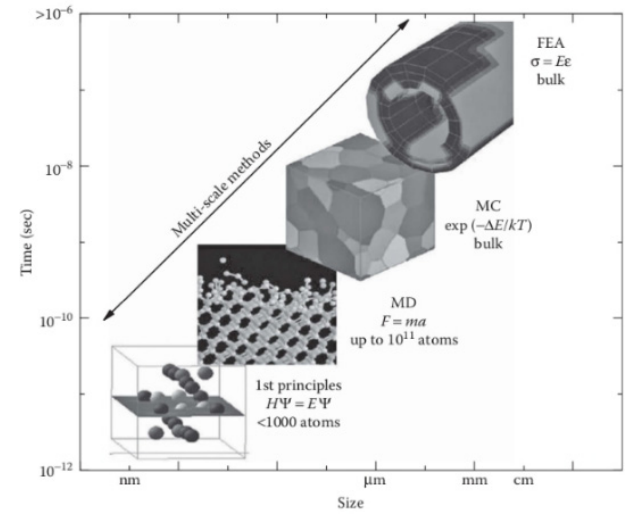

Figure 2. Typical methods in computational materials science in terms of size and time ${ }^{[21]}$.
Quantum Mechanics $(\mathrm{QM})^{[1,2,9,20,21]}$ method is based on the uncertainty principle, the motion behavior is described by the electron wave function, and the wave function is solved by the Schrödinger equation, $\widehat{\boldsymbol{H}} \boldsymbol{\Psi}=\boldsymbol{E} \boldsymbol{\Psi}(\widehat{H}$, Schrödinger operator; $\Psi$, electron wave function; $E$, energy). Ab Initio method is a method to solve Schrödinger equation, which does not use any empirical parameters, does not simplify the system, and strictly calculates all integrals $(<100$ atoms $)$, the calculations are accurate but slow. To calculate the macromolecular system with more atoms, in order to improve the calculation efficiency, some experimental value parameters are used instead of integral terms, so as to obtain a large number of semi-empirical calculation methods (such as CNDO, INDO, AM1 and PM3, etc.), among which AM1 and PM3 methods are the most widely used and have the highest calculation accuracy. In principle, the computational results of QM can essentially explain the conformation of molecules and the interactions between molecules. However, in the process of structural optimization, only local optimization is usually achieved, and the resulting optimal structure is related to the initial conformation. If insufficient conditions are considered for the initial conformation, the purpose of energy minimization is often not achieved. In a complex and huge system, the QM method is not applicable due to the large number of electrons $(>1000$ electrons).

Molecular Mechanics (MM) ${ }^{[1,2,9,20,21]}$ based on the Born-Oppenheimer approximation principle and classical Mechanics, calculate Molecular characteristics by Molecular force field. It is often used to calculate molecular equilibrium configuration and energy, such as the lowest energy configuration, configurational transition energy barrier and vibration frequency and other molecular thermodynamic properties. How to accurately use the molecular force field to restrain the molecules is the key to molecular mechanics, that is, what kind of force field to describe the molecular motion. The current common force fields include MM form force field, AMBER force field, CVFF force field, CHARMM force field, COMPASS and COMPASSII force field, etc. Molecular mechanics regards a molecule as a configuration composed of springs (chemical bonds) and balls (atoms) with different force constants. Its advantage is that it is simple and efficient to calculate the stable conformation and thermodynamic properties of large systems and complex molecules. However, different from $\mathrm{QM}$, the microscopic category involved in molecular mechanics cannot obtain the properties related to electron distribution.

Monte Carlo (MC) $)^{[1,2,9,20,21]}$ method is a non-quantum computing method, based on the probability distribution principle of statistical mechanics, to obtain statistical and thermodynamic data of the system by random walking of particles. The basic simulation is mainly based on random sampling. In a given ensemble, based on the known molecular potential energy function, the internal energy between particles is added, and the Metropolis sampling method of particles within the system for all kinds of "random walk"(displacement, rotation, and phase transfer, etc.), then the random configurations 
under the Boltzmann distribution is obtained when the microscopic particles in the ensemble tend to be balanced. The advantage is that the means is conceptually accessible and simple to conduct, because it simply lets the atoms jump randomly and select the lowest state of energy. It can balance any degree of freedom and is suitable for multi-dimensional and complex systems. However, because the particle displacement simulated by Monte Carlo is an artificial motion process, which does not represent the real motion trajectory of the particle, it only satisfies the Boltzmann distribution, so dynamic property simulation (such as transitive property) cannot be carried out, and MC method is only applicable to equilibrium simulation. The adsorption and diffusion of reservoir oil and gas in a specific geological environment is a random event, and $\mathrm{MC}$ can be used to study the mechanism, which can better simulate the adsorption/diffusion mechanism of oil/gas under complex geological conditions ${ }^{[22]}$.

Molecular Dynamics(MD $)^{[1,2,9,20,21]}$ method is based on Newton's law of motion. $\mathrm{MD}$ is the integral of Newton's equation of motion over time to gain the time evolution of the system, thereby obtaining the properties we are interested in. Given the initial position and velocity of each atom, and using the provided interatomic potentials, calculate the force on each atom. Then using that information, the initial position will advance to a lower energy state in a small time interval (called the time step $\Delta \mathrm{t}$ ), resulting in a new position, speed, etc. Finally, using these new data as input, the above steps are usually repeated, with more than thousands of time steps, until equilibrium is attained, and the system properties will not change over time (the process is shown in Figure. 3). During and after equilibrium, a variety of raw data stored for each or some time step, including atomic position and momentum, energy, force, etc. During and after the balance, various raw data stored for each or certain time steps, including atomic position and momentum, energy, force, etc., can be directly or indirectly used for statistical analysis of these data to calculate attributes, the dynamic and equilibrium statistics can be obtained at the same time, including basic thermodynamic, structural and mechanical properties, radial distribution function and diffusion coefficient of liquids, etc. Compared with $\mathrm{QM}$ and $\mathrm{MC}$, $\mathrm{MD}$ is very fast and accurate, so it can handle larger systems (tens of thousands of particles). However, some limitations of MD include the inability to obtain electromagnetic properties and the time scale is limited to nanoseconds, etc. Applying MD to the adsorption and diffusion of shale gas can not only gain the evolution history of shale gas over time, but also obtain physical quantities such as relative density and mean square displacement through statistical averages, further deepening the insight of underground conditions of shale $\operatorname{gas}^{[22]}$.

For detailed introduction of related and more molecular simulation techniques, please refer to related literature ${ }^{[1,2,9,20,21]}$, Researchers can choose the corresponding molecular simulation method according to their own research direction. It can be comprehensively applied, such as QM/MM combination method and
MC/MD "hybrid" method, so as to achieve the complementary advantages and disadvantages among algorithms and make the simulation accurate and efficient ${ }^{[11,23]}$.At present, the most widely used molecular simulation software includes LAMMPS, GROMACS, DL POLY, Materials Studio and so on. In the field of EOR engineering, molecular simulation is more and more widely used, such as the interaction mechanism between surfactants and crude oil, the design of oil displacement agent molecules and the analysis of oil and gas structure, etc ${ }^{[24,25-28]}$.

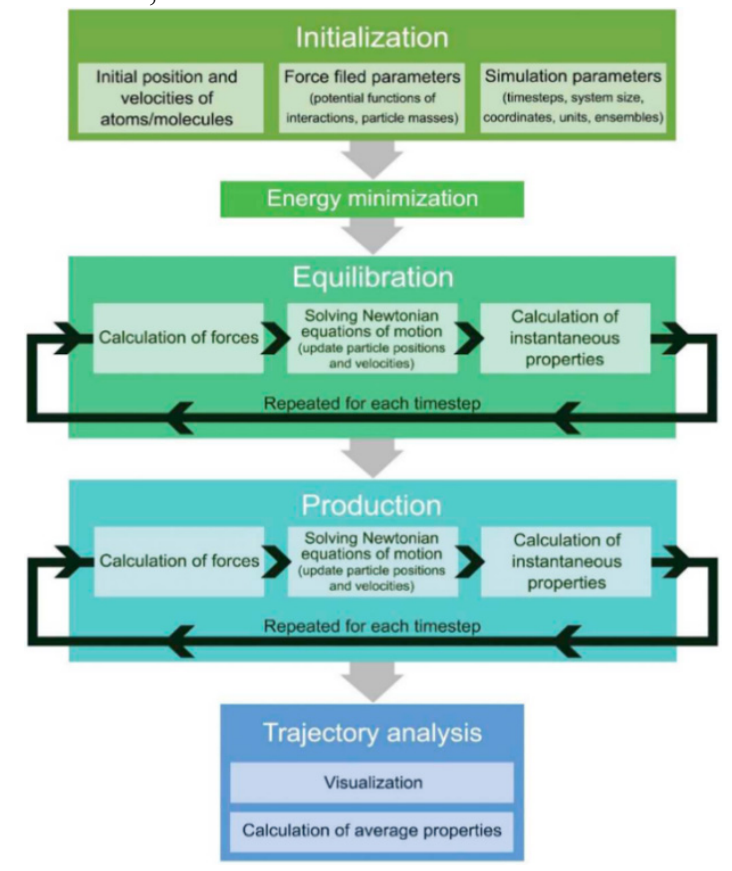

Fig 3. General workflow of MD simulation ${ }^{[29]}$.

\section{Study on the application of molecular simulation in EOR}

In the petroleum industry, experimental research and simulation have been used to find EOR methods. Molecular modeling is the preferred method in the study of replication under extreme conditions (such as high pressure and temperature), and it has been increasingly applied in EOR methods ${ }^{[30,31,32]}$. The application of molecular simulation techniques in some mainstream EOR methods is described below.

\subsection{Application of molecular simulation in polymer flooding}

\subsubsection{Analysis of polymer floodin mechanism}

Previous efforts are helpful to understand the mechanism of polymer flooding, but there is a shortage of insight into polymer flooding process at the atomistic level. The driving process of oil/gas trapped in a complex nano-scale environment is still unclear, such as oil droplets confinement to dead end of nanopores ${ }^{[33]}$.

SongKaoping Group ${ }^{[34]}$ in China was the first to describe the mechanism of improving the oil 
displacement efficiency of polymer by using the basic principles of molecular dynamics, and compared with the molecular force of water flooding, the viscoelasticity of polymer solution is the macroscopic manifestation of the collision force and friction force between polymer molecules and crude molecules, which can enhance the oil detachment efficiency. Fan et al. ${ }^{[41]}$ explored the mechanism of viscoelastic polymers to enhance oil recovery through MD simulations, and visualized the dynamic process of displacement of oil droplets trapped in nanopores from dead end. The research shows that the key factor for polymer flooding to perform well in recovery is the unique elastic properties of polymer molecules. Compared with simple water flooding, the main reason why polymer flooding improves microscopic oil dispalcement efficiency is the pulling effect mechanism. It is also show that polymers with longer chain tend to displace more residual oil with less injected PV(pore volume), obtaining higher microscopic oil recovery efficiency. The analyses of viscoelasticity of polymers showed that the longer the polymer chain length, the greater the polymer storage modulus, the longer the relaxation time of the polymer, and the stronger the elastic effect of the polymer (see Figure .4). This increase in elasticity causes the polymer to stretch more in the pore space and exerts a stronger pulling force the oil droplets in the dead ends, which is conducive to enhancing the oil displacement efficiency. The work proves that polymer flooding can enhance oil recovery under confined nanoscale. These findings are helpful to understand the microscopic oil displacement mechanism of polymer flooding and have guiding significance for the actual oil recovery process.
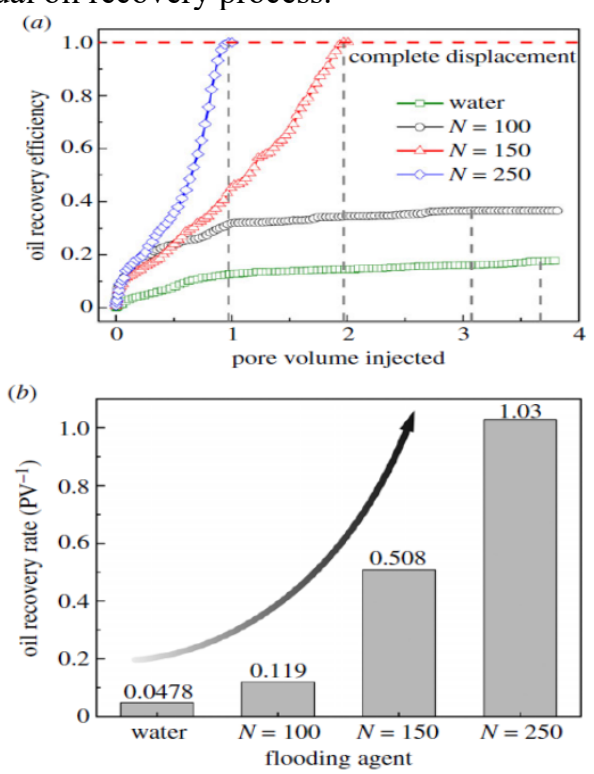

Figure 4. (a) Oil recovery efficiency during flooding process employing different flooding agents. Attaining the horizontal red dashed line means the whole oil droplet is displaced out of the dead end. The vertical grey dashed lines provide the corresponding injected PV when oil recovery efficiency attains a steady value. (b) Oil recovery rate obtained by different flooding agents ${ }^{[34]}$.

\subsubsection{Performance analysis and effect prediction of polymer flooding agent}

Chen et al. ${ }^{[35]}$ used MD to study the effect of salinity on the viscosity of polyacrylamide oil displacement agents, on this basis, Li et al. ${ }^{[36]}$ adopted the same simulation technique to study the influence of hydrated montmorillonite on the viscosity of polyacrylamide with changing temperature and shear rate, and the results of simulation and experiment are consistent. MD simulation is an effective tool for studying the conformation and viscosity properties of polymer solutions, which can provide useful information for the synthesis of polyacrylamide derivatives with ideal solution properties, however, experiments cannot provide the molecular configurations and their changes at the microscopic level ${ }^{[35]}$. Ni et al. ${ }^{[37]}$ explored the influence of salt ions on the structure of anionic polyelectrolyte chain using $\mathrm{MM} / \mathrm{MD}$ combination, and provided a scientific and reasonable explanation for the separation or settlement of polyanions in salt solution, which can be used to improve the new oil displacement agent; The atomic model of crosslinked polyacrylamide network was established by $\mathrm{MM}$ and $\mathrm{MD}$, and the combination of experimental characterization and molecular simulation is a new method to study the mechanism of crosslinked reaction, the resol crosslinked polyacrylamide hydrogel studied has good temperature and salt resistance performance and can be applied as a chemical oil-displacing agent to improve the recovery ${ }^{[38]}$.

\subsection{Application of molecular simulation in surfactant flooding}

Although a lot of researches for the macroscopic dynamics of oil recovery process have provided great insights, owing to the different nature of reservoir rocks and the complex components of crude oil, it is a challenging research subject to understand the molecular mechanisms of oil detachment by chemical flooding. Therefore, the investigation of nano-scale dynamics in the process of surfactant flooding has important theoretical significance for the design of surfactant molecules with high oil recovery efficiency ${ }^{[39]}$.

Jiang Rongjun et al. ${ }^{[40]}$ used MD to explore the behavior of surfactants on the oil-water interface, discussed the oil washing performance of various types of surfactants, and obtained the surfactant washing efficiency as Betaine $>$ sodium dodecyl benzene sulfonate $(\mathrm{SDBS})>$ nonylphenol polyoxyethylene ether(NPE)> dodecyl trimethylammonium bromide(DTAB), simulation results are in agreement with the pratical displacement, explaining the law of washing oil by different surfactants at the molecular level, at the same time, it can analyze and compare the law of washing oil of nanoparticles and their modified substances.

Tang et al. ${ }^{[41]}$ utilized MD simulation to explore the process of ionic surfactant solution driven oil dispalcement in $\mathrm{SiO}_{2}$ nanochannels model (see Figure 5), which can be illustrated by a three-stage process, including the formation and delivery of surfactant micelles, the disintegration-spread and migration of 
surfactant micelles on the surface of oil aggregate, and the deformation-to-detachment of oil molecular aggregate; Under the action of water flow, the surfactant molecules migrate to the rear bottom of the oil aggregate, allowing the water molecules to occupy the surface sites of the oil molecules and cause the oil molecules to detach; The SDBS exhibited a better oil displacement efficiency than DTAB (consistent with the results of literature [40]), this is because the $-\mathrm{SO}^{-}$group in $\mathrm{SDBS}$ is more attractive to water molecules, which leads to faster migration of SDBS on the surface of oil aggregate, thus obtaining higher deformation-detachment speed of oil molecules, and the simulation results are useful to understand the interaction between surfactant solution and crude oil and provide some fresh understanding for the process of surfactant flooding to enhance oil recovery.

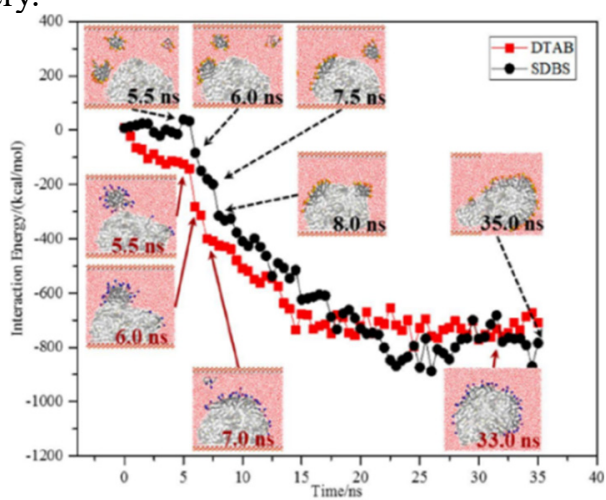

Figure. 5 The snapshots of the surfactant/water/oil at different time stages ${ }^{[41]}$

\subsection{Application of molecular simulation in $\mathrm{CO}_{2}$ and $\mathrm{N}_{2}$ flooding}

Understanding the interfacial phenomenon or behaviour of crude oil, water and injected $\mathrm{CO}_{2}$ is very important to improve $\mathrm{CO}_{2}$-EOR and $\mathrm{CO}_{2}$ capture and sequestration (CCS) process, and it is difficult to elucidate its interfacial properties from experiments, at present, researches on the ternary $\mathrm{CO}_{2}$-water-oil system are very limited, particularly on the microscopic behaviour of $\mathrm{CO}_{2}$ distribution at the interface, and the structure and dynamic properties of the water-oil interface have not been stated ${ }^{[42]}$. According to the investigation, there is no atomic-level research on the interaction between small gas molecule and coal macromolecule has been reported, employing $\mathrm{MD}$ techniques simulate the diffusion and adsorption properties of gases $\left(\mathrm{CO}_{2}, \mathrm{~N}_{2}, \mathrm{CH}_{4}\right)$ in coal, in order to elucidate the details of adsorption and diffusion and demonstrate the feasibility of using this simulation technique in the field of coalbed methane, to provide useful insight and guideline for enhancing coal bed methane recovery (ECBM) and CCS engineering ${ }^{[43]}$.

\subsection{1 conventional petroleum}

Fang et al. ${ }^{[44]}$ explored the behavior displacement and adsorption of oil on silica surface by $\mathrm{CO}_{2}$ at different temperatures $(303 \mathrm{~K}, 323 \mathrm{~K}, 343 \mathrm{~K}, 363 \mathrm{~K}$ and $383 \mathrm{~K})$ by adopting MD, at the temperature of $343 \mathrm{~K}$, the adsorption oil has the highest diffusion capacity and the best detachment performance, it provides a molecular-scale insight of oil displacement (IFT) between oil and water. which is conducive to EOR. The data obtained basically coincides with the experimental data ${ }^{[45]}$, as shown in Figure .6

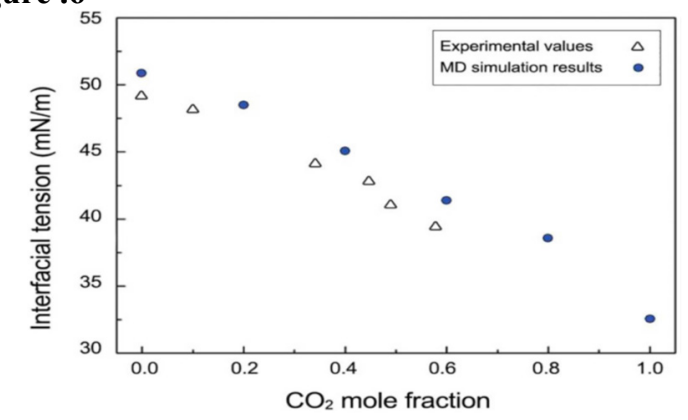

Fig 6 Comparison of MD simulation results on IFT of water/oil as a function of $\mathrm{CO}_{2}$ mole fraction at $313 \mathrm{~K}$ and $10 \mathrm{MPa}$ with experimental values. A linear decrease is observed with increasing $\mathrm{CO}_{2}$ mole fraction ${ }^{[37,42]}$.

Fang et al. ${ }^{[46]}$ studied the mechanism of $\mathrm{scCO}_{2}$ selective extraction causing the reservoir wettability alteration through MD simulation (see Figure .7). The results demonstrate that $\mathrm{scCO}_{2}$ can disrupt the structure of the oil film and facilitate the formation of molecular channels. Then $\mathrm{scCO}_{2}$ can strip the non-polar components of the crude oil from the surface of reservoir, leaving the polar components of the crude oil on the surface of reservoir (see Figure .8). This selective extraction behaviour of $\mathrm{scCO}_{2}$ is the key to the separation of non-polar and polar components in crude oil. Therefore, after $\mathrm{CO}_{2}$ injection, the wettability of reservoir surface changes from hydrophobic to hydrophilic, and the spontaneous imbibition capacity of injected water is significantly improved. This research has guiding significance for $\mathrm{CO}_{2}$-EOR; It is of great importance to optimize the oil recovery process during water-alternating-gas injection (WAG) cycles, which is also confirmed below (2.6.2).

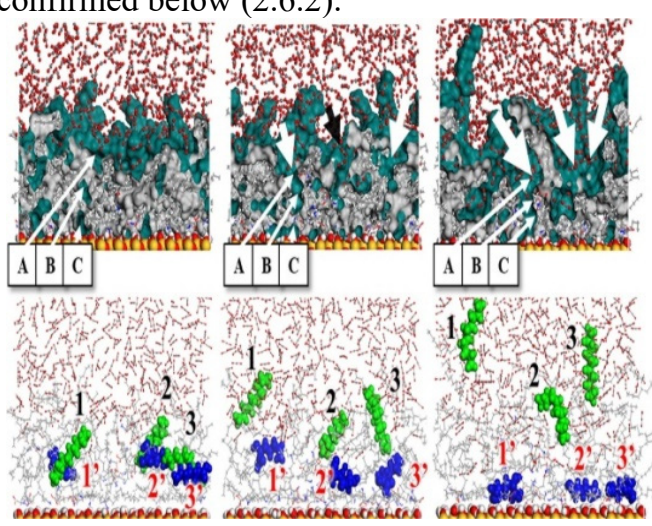

Fig 7 Snapshots of water droplets adsorbed on oil reservoir rocks (a) with apolar and polar components (before $\mathrm{CO} 2$ injection) and (b) after extraction of the apolar components (after $\mathrm{CO} 2$ injection). Notes: the yellow lines show contacts, and the atoms are colored as follows: $\mathrm{O}$, red; $\mathrm{C}$, gray; $\mathrm{N}$, light blue; $\mathrm{H}$, white ${ }^{[46]}$. 


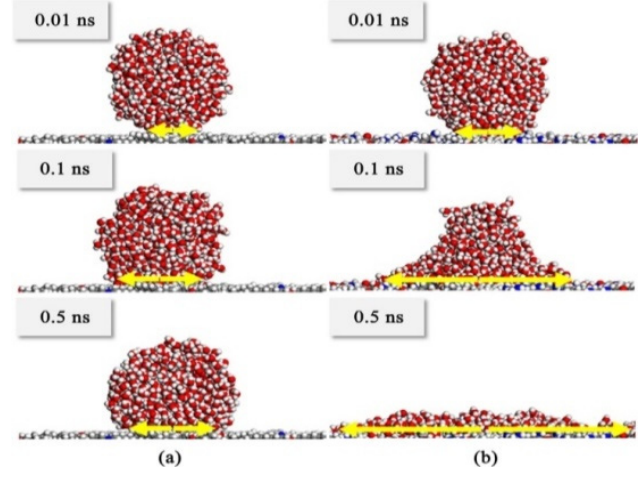

Fig 8. (up) Interface snapshots between $\mathrm{scCO} 2$ and oil film at $0.01 \mathrm{~ns}, 0.02 \mathrm{~ns}$ and $0.05 \mathrm{~ns}$. Notes: free space in oil film, deep green; spatial volume occupied by oil molecules, silver. (down) Representative snapshots of the system in oil film. Three pairs of polar (lysine) and apolar (decane) molecules are highlighted in blue and green for clarity ${ }^{[46]}$.

\subsubsection{Shale oil and gas}

Fang et al. ${ }^{[47]}$ explored the mechanism of $\mathrm{CO}_{2}$ extraction of hydrocarbon in rough surface, and also proved that the solubility of $\mathrm{CO}_{2}$ plays a key role in enhancing the recovery rate of tight oil in Nano pores, indicating that $\mathrm{CO}_{2}$ injection depends largely on the structural characteristics of the reservoir, thereby providing a quantitative insight of the mechanism of $\mathrm{CO}_{2}$ extraction of crude oil in the rough surface.

Huang et al. explored the competitive adsorption of $\mathrm{CO} 2 / \mathrm{CH} 4$ in realistic Kerogen model through molecular simulation technology, low reservoir pressure is conducive to the replacement of $\mathrm{CH}_{4}$ by $\mathrm{CO}_{2}$ in shale kerogen, the stability of $\mathrm{CO}_{2}$ adsorption decreases with rising the molar ratio of $\mathrm{CO}_{2}$ in reservoir, increasing the reservoir moisture content can improve the performance of $\mathrm{CO}_{2}$ sequestration with enhanced $\mathrm{CH}_{4}$ recovery (CS-EGR), and the sulfur-containing groups are the preferred adsorption site for $\mathrm{CH}_{4}$, while the nitrogen- and oxygen-containing groups are the preferred adsorption sites for $\mathrm{CO}_{2}$, thus the understanding on the molecular scale of $\mathrm{CO}_{2}$ exploitation of shale gas is enhanced, which can provide practical guidances for the optimization of CS-EGR in shale gas reservoirs ${ }^{[17]}$.

Xue et al. ${ }^{[48]}$ used MD to simulate the migration process of oil in silica, calcite, and dolomite shale channels driven by $\mathrm{N}_{2}$, and found that there is obvious oil transportation in silica and calcite, while the oil molecules in the dolomite channel keeped basically its position. The different oil transportation properties are attributed to the different interaction energy between oil molecules and shale channels, dolomite $>$ calcite $>$ silica.

\subsubsection{CBM(Coalbed Methane)}

$\mathrm{Hu}$ et al. ${ }^{[52]}$ studied and compared the diffusion and adsorption behaviors of $\mathrm{CH}_{4}$ and $\mathrm{CO}_{2}$ in coal through molecular simulation, and the computed $\mathrm{CO}_{2}$ diffusion coefficient was about $10^{-9} \mathrm{~m}^{2} / \mathrm{s}$, which is quite near to the experimental result, $\mathrm{CH}_{4}$ diffused slightly faster than $\mathrm{CO}_{2}$ and sorption heat $\mathrm{CO}_{2}>\mathrm{CH}_{4}$, which may be the reason why $\mathrm{CO}_{2}$ can displace the adsorbed $\mathrm{CH}_{4}$ gas in coal bed, thus improving the recovery efficiency of $\mathrm{CH}_{4}$.

Zhao et al ${ }^{[52]}$ computed the diffusion coefficients of $\mathrm{CH}_{4}$ and $\mathrm{CO}_{2}$, and concluded that the transport diffusion coefficients were consistent with the experimental data, $\mathrm{CO}_{2}$ and $\mathrm{CH}_{4}$ mainly diffuse in coal micropores, and the diffusion rate $\mathrm{CO}_{2}>\mathrm{CH}_{4}$, three different diffusion coefficients increase with temperature; with increasing pressure, the transport diffusion coefficient first increases, then reaches the peak, and then decreases gradually; at relatively low pressure, coal matrix swelling appears to be negligible and the dominant migration mechanism is the surface diffusion; with the increase of pressure, the swelling speed of coal is accelerated, the diffusion activation energies is enhanced, and the configurational diffusion is gradually dominant; the peak pressure of $\mathrm{CO}_{2}$ (produces peak diffusion coefficient, $\mathrm{CO}_{2}>\mathrm{CH}_{4}$ ) less than $\mathrm{CH}_{4}$.

The above calculation of diffusion coefficients does not take into account the mutual diffusion of gases, Hu et al. ${ }^{[49]}$ explored the self-diffusion and mutual diffusion of $\mathrm{CO}_{2}-\mathrm{CH}_{4}$ mixtures by molecular simulation for the first time, and founded that the self-diffusion coefficients (SDC) of $\mathrm{CO}_{2}$ and $\mathrm{CH}_{4}$ decreased with the increase of gas concentration, but increased with the increase of temperature.

\subsection{Application of molecular simulation in foam flooding}

In order to solve the low viscosity and density of gas in gas injection flooding, the gas and surfactant are injected together to produce foam, and in an attempt to maintain the stability of the foam, nanoparticles and polymers are added as stabilizers. In the foam, the continuous phase forms thin liquid films, and the stability of the foam basically depends on the stability of these films, Chen et al. ${ }^{[50]}$ adopted MD method to investigate the effect of the presence of $\mathrm{NaCl}$ in the SDS foam system on the electrostatic properties of NBF (Newtonian black film, film $<5 \mathrm{~nm}$ ), and found that the addition of salt does not change the stable thickness of NBF.

$\mathrm{Wu}$ et al. ${ }^{[51]}$ employed MD method to explore the effect of polymer (polyacrylamide, PAM) concentration and hydrolysis degree on the stability of SDS foam system, the results suggest that the interaction between PAM and SDS increases the membrane elasticity, and with increasing PAM concentration, the number of water molecules around the SDS head group increases, and when the hydrolysis degree of HPAM is within $20 \sim 30 \%$, the SDS foam system has strong water retention and the best foam stability.

\subsection{Application of molecular simulation in combined flooding (polymer- surfactant)}

The polymer and surfactant compounding system has been widely used in petroleum field. Understanding the interaction mechanism from a microscopic view is the key to guiding the production practice. With the development of molecular simulation technology, the 
study of the interaction mechanism between polymers and surfactants at the molecular level has been widely carried out, and a large number of research results have been obtained ${ }^{[52]}$.

Cao Xulong ${ }^{[53]}$ adopted DPD simulation and physical experiment model to design the formula of polymersurfactant binary combination flooding, and achieved $18.1 \%$ enhanced oil recovery effect. Aiming at exploring the cooperative oil displacement mechanism of combined flooding, on the basis of Cao Xulong's research, Ren Qiang et al. ${ }^{[54]}$ adopted DPD approach to study the interfacial phenomenon of the binary combined flooding system, and investigated the interaction mechanism between oil-displacing agent molecules and oil molecules at the molecular level.

$\mathrm{Li}$ et al. ${ }^{[55]}$ investigated the effects of surfactant concentration, polymer concentration and oil/water ratio on structural properties and interfacial properties through DPD simulation. In the presence of polymers, the interface is supersaturated at a low surfactant concentration. The formation of polymer/surfactant complexes is conducive to the reduction of oil-water IFT. Therefore, the successful application of DPD method to study the structure and interfacial properties of surfactants at the oil/water interface, particularly the influence of polymers, has a great significance for the beneficial application of surfactants.

\subsection{Application of molecular simulation in new oil displacement technology}

\subsubsection{Nanoparticle flooding}

Due to the low viscosity of $\mathrm{scCO}_{2}$, the macroscopic cleaning efficiency is reduced. Aiming at solving this problem, Mahdavi et al. ${ }^{[56]}$ used MD to explore the effect of the morphology of alumina nanoparticles $\left(\mathrm{Al}_{2} \mathrm{O}_{3} \mathrm{NP}\right)$ on the rheological properties of $\mathrm{scCO}_{2}$ (such as viscosity and self-diffusion coefficient), and obtained a good viscosity enhancement effect. Soleimani et al. ${ }^{[57]}$ employed $\mathrm{MC}$ to study the adsorption of $\mathrm{ZnO}$ nanoparticles (NPs) on the surface of calcite (111), the adsorption of $\mathrm{ZnO}$ on the surface of rocks changed the wettability of rocks, thus reducing capillary force and improving the recovery rate. Sagala et al. ${ }^{[74]}$ carried out graft modeling for nanopyroxene through molecular simulation (see Figure .9), and designed oil displacement agent for core displacement experiment under the condition of applying reservoir temperature, and achieved an improved recovery rate of $10.57 \%$ after water injection, meeting the requirements of molecular structure design.
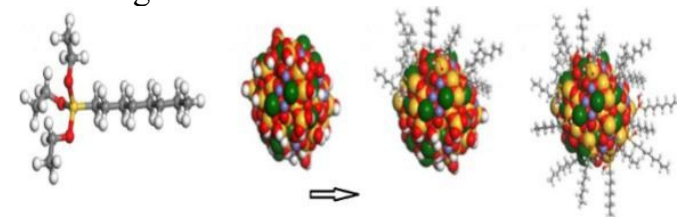

Fig 9. Modeling process of graft modification of nanopyroxene nanoparticle, modified after ${ }^{[36]}$.
Liang et al. ${ }^{[58]}$ designed three kinds of modified nanoparticles grafted with hydrophilic chain (NP1), hydrophobic chain (NP2) and mixed hydrophilic and hydrophobic chain (NP3) for enhanced oil recovery. The detachment performance of the equilibrium adsorption configuration and dynamic driving process was studied by molecular dynamics simulation. The simulation results show that, compared with pure water, the three kinds of modified grafted nanoparticle solutions can promote oil droplet separation, and NP3 has the best ability to enhance oil recovery.

\subsubsection{CO2 WAG injection}

Yan et al. ${ }^{[59]}$ investigated the displacing process of adsorbed oil layer in nanoslit employing $\mathrm{scCO}_{2}$, water and $\mathrm{CO}_{2}$ WAG injection through non-equilibrium $\mathrm{MD}$ simulation, and revealed the EOR mechanism of $\mathrm{CO}_{2}$ WAG (see Figure 10.). The simulation results show that $\mathrm{scCO} 2$ and water play different roles in the process of displacement. The $\mathrm{scCO}_{2}$ slug is bound to dissolve the non-polar oil components, and the water slug compelled the miscible decane $/ \mathrm{scCO}_{2}$ slug out of the nanopore. In addition, $\mathrm{scCO}_{2}$ slug and water slug have excellent synergetic phenomena such as high dissolution efficiency, easy discharging of residual water, widening of fluid channel, and good detachment effect, so that $\mathrm{CO}_{2}$ WAG injection is characterized by low injection pressure and high recovery rate. This work gives an atomic level insight into the replacement process of $\mathrm{CO}_{2} \mathrm{WAG}$, and the research results are supportive to understand the nature of some engineering phenomena, and give reference for the optimization of $\mathrm{CO}_{2}$ WAG injection project.
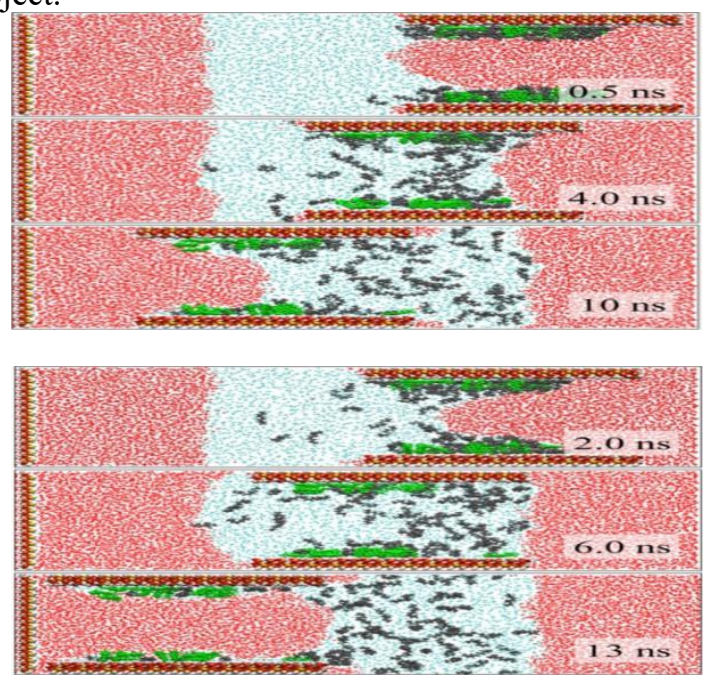

Fig 10. Snapshots of displacement process of adsorbed oil layer in $\mathrm{CO} 2$ WAG injection. (CO2, cyan; water s red ; decane, gray; asphaltene, green. $)^{[60]}$.

\subsubsection{Low salinity waterflooding (LSW)}

Yang Jie et al. ${ }^{[77]}$ first carried out laboratory experiments to obtain macroscopic characterization, and then combined with molecular simulation to analyze the mechanism of wettability alteration on sandstone surface 
by $\mathrm{pH}$ increase of imbibing brine. Sedghi et al. ${ }^{[61]}$ conducted $\mathrm{MD}$ to explore the effect of wall-fluid interactions (i.e. nano-confinement effect) on threshold capillary pressure of crude oil-water/brine displacement in the mesopore of angular calcite.

Zhao et al. ${ }^{[62]}$ employed classical equilibrium MD (EMD) simulation to study the influence of water salinity in changing the wettability of narrow nanopores for three types of surfaces of calcite (neutral non-polar surfaces, positively and negatively charged polar surfaces ); moreover, non-equilibrium MD (NEMD) indicates the effect of wettability on the oil transport properties in nanopores at different salt concentrations of $\mathrm{NaCl}(0.20 \mathrm{M}$, $0.50 \mathrm{M}$ and $1.00 \mathrm{M})$. The results suggest that rising water salinity has little influence on the wettability of nanopores composed of neutral calcite surface.

\subsection{Application of molecular simulation in microemulsion flooding}

Microemulsion liquid flooding is regarded as a relatively advanced oil displacement technology in tertiary oil recovery. It forms a microemulsion by adding surfactants and co-surfactants in the oil-water system to wash the oil. Larson et al. ${ }^{[63]}$ and Gunn et al. ${ }^{[64]}$ simulated the equilibrium properties of microemulsion phase formed by surfactant - water - oil by MC method, and computed the diffusion coefficient and surface tension, etc. and the results were in good qualitative and even quantitative agreement with the experimental data.

$\mathrm{Wu}$ Chuan et al. ${ }^{[65]}$ explored the effect of microemulsion nano-nickel on the upgrading and viscosity reduction of extra-heavy oil, the mechanism of upgrading and viscosity-reducing of extra-heavy oil was investigated by experiment and MD simulation. The results suggested that after the upgrading and viscosity reduction reaction, the viscosity decreased significantly; the size of gum and asphaltene molecules is greatly reduced, and the effect of reducing the molecular weight of asphaltene is better; the modified viscosity reducer can contact the asphaltene in the discoid centronucleus of condensed aromatics as a heavy component of extra-heavy oil, and destroy the stable discoid aromatic ring structure, improve the aggregation status of asphaltenes, and enhance the stabilizing effect of gum molecules on asphaltene.

\section{Future prospects of molecular simulation in exploration and development of oil-gas}

Molecular simulation technology is still in the development stage in reservoir analysis. There are several aspects need to improve, that requiring further study to make this technology considered as a commonly used tool in the oil-gas industry. Including the use of molecular simulation to design generally acceptable models and procedures to represent complex reservoir fluids and heterogeneous porous media, develop fluid and rock property prediction toolboxes, study cemented formations and production paths in reservoir rocks, and further improve the understanding of the basic mechanism of EOR and the results predicted, especially for less mature EOR methods. At present, there are still some directions that have not been studied/to be studied or less studied, such as the ternary combination flooding and other binary complex flooding simulations that have not been mentioned in the paper, and the simulations of polymer flooding and low-salinity water flooding are rarely carried out, so then enriching the mechanism of oil recovery and build reliable models in the future. It can also be used in the study of fracturing fluids and the mechanism of profile control and water plugging agent. Basic research on the application of new clean fracturing fluids, nano oil displacement agents, foam oil displacement systems and intelligent control and flooding systems was carried out to realize the directional design of function-oriented self-assembly systems based on molecular simulation; the research on the adsorption, desorption, diffusion, migration and mass transfer of oil and gas in nano and nano-micro pores reveals the microscopic mechanism and chief controlling factors of oil/gas occurrence and migration, and explores the effective docking between microscopic and macroscopic, thus providing a theoretical basis for the development of shale and tight oil/gas. Research on the adsorption and residence rule of $\mathrm{CO}_{2}$ in the reservoir, research and development for the chemical system of $\mathrm{CO}_{2}$-driven mixing and thickening, and design of the surfactant $/ \mathrm{scCO}_{2}$ fracturing system are carried out to improve the theoretical and technical support for EOR and reservoir reconstruction.

\section{Conclusions}

(1) The traditional research methods have been difficult to analyze the complex formation conditions as the difficulty of reservoir exploration and the increase of reservoir exploitation depth. It is therefore vital to develop reliable approaches for the dependable prediction of fluid and rock characteristics and connected flow-related phenomena. Molecular simulation is an advanced method that has currently attracted great focus on reservoir research owing to its ability to study physical phenomena at the atomic and molecular scale.

(2) Molecular simulation can be used to study a large number of fluid and rock properties, interactions and related concepts. The application of molecular simulation provides a unique choice for reservoir analysis. Computer experiments can be carried out quickly, economically and risk-free to explore the properties of some complex phenomena at the atomic and molecular levels.

(3) The application of this technology will build a bridge between theory, experiment and simulation, and prove each other. The model designed by it is more practical, qualitative to quantitative than the experimental model.

(4) Due to the complexity of oil composition and heterogeneity of reservoir, there are some differences between the real system and the simulation system. How to reduce this difference is an urgent problem for 
researchers.

\section{Acknowledgments}

Supported by National Natural Science Foundation of China (51874241)

\section{References}

1. Chen Z L, Xu W R, Tang L D. Theory and Practice of Molecular Modeling[M]. Beijing: Chemical Industry Press, 2007.

2. Yang Xiaozhen. molecular modelling and macromolecular material[M]. Beijing: Science Press, 2002.

3. Hou Yanbo, Ren Qiang, Dai Zhenyu, Zhou Han. Interaction mechanism between surfactant molecules and oil-water interface molecules [J]. Acta petroleum Sinica (petroleum processing), 2018,34 (01): 108-114.

4. Hou Yanbo, Ren Qiang. Mesoscopic simulation of surfactant oil water interface [J]. Acta petrologica Sinica (petroleum processing), 2019,35 (01): 91-98.

5. Yayun Zhang, Shuyang Gao, Xiaoyu Du, et al. Molecular dynamics simulation of strength weakening mechanism of deep shale[J]. Journal of Petroleum Science and Engineering, 2019, 181.

6. song Jinbo, Chai Yongming, Yan Chunru, Zheng duo. Mechanism of relative permeability regulator [J]. Petroleum geology and oil recovery, 2013,20 (01): $104-106+110+118$.

7. Junfang Zhang, Michael B. Clennell, Keyu Liu, David N. Dewhurst, Marina Pervukhina, Neil Sherwood. Molecular dynamics study of CO2 sorption and transport properties in coal[J]. Fuel,2016,177.

8. Geng tie, Zhao Chunhua, Liu Xuejing, Su long, Zheng Liqiang, sun Jichao. Aggregation behavior of surfactant molecules at oil / water interface: progress in molecular simulation [J]. Daily chemical industry, 2019,49 (08): 537-544.

9. Chen $\mathrm{M}$ B. Computational chemistry- from theoretical chemistry to molecular simulation[M]. Beijing: Science Press, 2009.

10. Ren Wen-po, Chen Hong-gang, Yang Chao-he, et al. Application of molecular simulation in density characterization and structure validation of heavy oil fractions[J]. CIESC Journal, 2009, 60(08): 1883-1888.

11. Qu Huimin, Ding Zifeng, men Haiying, Wang Ning, Wang Peng, Wei Liangxia. Molecular simulation, synthesis and performance evaluation of molecular membrane injection enhancer [J]. Oilfield chemistry, 2017,34 (03): 463-468.

12. Qu Jiali, Li Xiangyuan, Chen Jue, et al. Application of molecular simulation technology in polymer science experiments[J]. Polymer Bulletin, 2017(05): 69-72.
13. Chu Zhang, Shangbin Chen, Yu Liu, et al. Mechanism of methane adsorption on groove space in organic matter surface[J]. Molecular Simulation, 2019,45(3).

14. Shi J, Gong L, Sun S, et al. Competitive adsorption phenomenon in shale gas displacement processes[J]. RSC Advances, 2019, 9(44): 25326-25335.

15. Haixiang $\mathrm{Hu}$, Lei Du, Yanfei Xing, Xiaochun Li. Detailed study on self- and multicomponent diffusion of $\mathrm{CO} 2-\mathrm{CH} 4$ gas mixture in coal by molecular simulation[J]. Fuel, 2017, 187.

16. Huang, L., Ning, Z., Li, H., et al. Molecular Simulation of $\mathrm{CO} 2$ Sequestration and Enhanced Gas Recovery in Gas Rich Shale: An Insight Based on Realistic Kerogen Model[J]. Society of Petroleum Engineers, 2017.

17. Geng Tie, Zhao Chun-hua, Liu Xue-jing, et al. Molecular simulations for aggregation behavior of surfactant molecules at oil/water interface[J].

18. Aimoli C G, Maginn E J, Abreu C R, et al. Force field comparison and thermodynamic property calculation of supercritical $\mathrm{CO} 2$ and $\mathrm{CH} 4$ using molecular dynamics simulations[J]. Fluid Phase Equilibria, 2014: 80-90.

19. Qin Zhang, Feng Liang, Zhenglian Pang, et al. Lower threshold of pore-throat diameter for the shale gas reservoir: Experimental and molecular simulation study[J]. Journal of Petroleum Science and Engineering, 2019, 173.

20. Cao bin, Gao Jinsen, $\mathrm{Xu}$ Chunming. The Applications of Molecular Simulation Technology in the Fields of Petroleum[J]. Progress in Chemistry, 2004(02): 291-298.

21. June Gunn Lee. Computational Materials Science: An Introduction[M]. Boca Raton: CRC Press, 2016.

22. He Yingjie, Yang Yang, Zhang Tingshan, et al. Molecular simulation of shale gas adsorption in graphite slit-pores [J]. Lithologic Reservoirs, 2016, 28(06): 88-94.

23. Junfang Zhang, Michael B. Clennell, Keyu Liu, David N. Dewhurst, Marina Pervukhina, Neil Sherwood. Molecular dynamics study of $\mathrm{CO} 2$ sorption and transport properties in coal[J]. Fuel, 2016, 177.

24. Ungerer P, Yiannourakou M, Mavromaras A, et al. Compositional Modeling of Crude Oils Using C10 C36 Properties Generated by Molecular Simulation[J]. Energy \& Fuels, 2019, 33(4): 2967-2980.

25. Ponsjimenez M, Cartasrosado R, Martinezmagadan J $\mathrm{M}$, et al. Theoretical and experimental insights on the true impact of C12TAC cationic surfactant in enhanced oil recovery for heavy oil carbonate reservoirs $[\mathrm{J}]$. Colloids and Surfaces A: Physicochemical and Engineering Aspects, 2014, 455(455): 76-91.

26. Jie Zhong, Pan Wang, Yang Zhang, et al. Adsorption mechanism of oil components on water-wet mineral 
surface: A molecular dynamics simulation study[J]. Energy, 2013, 59.

27. Zhang Hongyu, Wang Yanyan, Tao Guoqiang, et al. Coarse Grained Molecular Mechanics (MM) /Molecular Dynamics (MD) Force Field for Petroleum Chemistry:I.Coarse Grained Model for Alkanes in Petroleum[J]. Acta Chimica Sinica, 2011, 69(17): 2053-2062.

28. Sagala F, Montoya T, Hethnawi A, et al. Nanopyroxene-Based Nanofluids for Enhanced Oil Recovery in Sandstone Cores at Reservoir Temperature[J]. Energy \& Fuels, 2019, 33(2): 877-890.

29. Masoud Seyyedattar, Sohrab Zendehboudi, Stephen Butt. Molecular dynamics simulations in reservoir analysis of offshore petroleum reserves: A systematic review of theory and applications[J]. Earth-Science Reviews, 2019, 192.

30. Liu B, Shi J, Sun B, et al. Molecular dynamics simulation on volume swelling of $\mathrm{CO} 2$-alkane system[J]. Fuel, 2015: 194-201.

31. Makimura, D., et al. Application of molecular simulations to CO2-enhanced oil recovery: phase equilibria and interfacial phenomena[J]. SPE, 2013, 18 (2), 319-330.

32. Zhang J, Pan Z, Liu K, et al. Molecular Simulation of $\mathrm{CO} 2$ Solubility and Its Effect on Octane Swelling[J]. Energy \& Fuels, 2013, 27(5): 2741-2747.

33. Fan Jing Cun, Wang Feng Chao, Chen Jie, et al. Molecular mechanism of viscoelastic polymer enhanced oil recovery in nanopores[J]. Royal Society open science, 2018, 5(6).

34. Song Kaoping, Yang Erlong, Wang Jinmei, et al. Mechanism of enhancing oil displacement efficiency by polymer flooding and driving effectiveness analysis[J]. Acta Petrolei Sinica, 2004(03): 71-74.

35. Chen P, Yao L, Liu Y, et al. Experimental and theoretical study of dilute polyacrylamide solutions: effect of salt concentration[J]. Journal of Molecular Modeling, 2012, 18(7): 3153-3160.

36. LI Wenzhuo, WANG Jianlong, XU Dingjia. Molecular Simulations of the Effect of Hydrated Montmorillonite on the Viscosity of Polyacrylamide under Confined Shear[J]. Journal of Wuhan University of Technology (Materials Science Edition), 2015, 30(03): 556-561.

37. Ni T, Huang G, Gao P, et al. Molecular Simulation of Salt Ion Effect on Anionic Polyelectrolyte Chain[J]. Journal of Macromolecular Science, Part B, 2012, 51(1): 60-69.

38. Tao Ni, Guang-Su Huang, Jing Zheng, et al. Research on the crosslinking mechanism of polyacrylamide/resol using molecular simulation and X-ray photoelectron spectroscopy[J]. Polymer Journal, 2010, 42(5).

39. Tang J, Qu Z, Luo J, et al. Molecular Dynamics Simulations of the Oil-Detachment from the
Hydroxylated Silica Surface: Effects of Surfactants, Electrostatic Interactions, and Water Flows on the Water Molecular Channel Formation[J]. The journal of physical chemistry. B, 2018, 122(6).

40. JIANG Rongjun, LUO Jianhui, BAI Ruibing, et al. Molecular Dynamics Simulation on Behavior of Common Surfactants at the Oil/Water Interface in Complex Systems[J]. Chemical Journal of Chinese Universities, 2017, 38(10): 1804-1812.

41. Tang X, Xiao S, Lei Q, et al. Molecular Dynamics Simulation of Surfactant Flooding Driven Oil-Detachment in Nano-Silica Channels[J].J Phys Chem B. 2019, 123(1): 277-288.

42. Liu B, Shi J, Wang M, et al. Reduction in interfacial tension of water-oil interface by supercritical $\mathrm{CO} 2$ in enhanced oil recovery processes studied with molecular dynamics simulation[J]. Journal of Supercritical Fluids, 2016: 171-178.

43. Haixiang $\mathrm{Hu}$, Xiaochun $\mathrm{Li}$, Zhiming Fang, et al. Small-molecule gas sorption and diffusion in coal: Molecular simulation[J]. Energy, 2010, 35(7).

44. Timing Fang, Muhan Wang, Chao Wang, et al. Oil detachment mechanism in $\mathrm{CO} 2$ flooding from silica surface: Molecular dynamics simulation[J]. Chemical Engineering Science, 2017, 164.

45. Raveendran P, Ikushima Y, Wallen S L, et al. Polar Attributes of Supercritical Carbon Dioxide[J]. Accounts of Chemical Research, 2005, 38(6): 478-485.

46. Timing Fang, Junqin Shi, Xiaoli Sun, et al. Supercritical $\mathrm{CO} 2$ selective extraction inducing wettability alteration of oil reservoir[J]. The Journal of Supercritical Fluids, 2016, 113.

47. Timing Fang, Yingnan Zhang, Rui Ma, et al. Oil extraction mechanism in $\mathrm{CO} 2$ flooding from rough surface: Molecular dynamics simulation[J]. Applied Surface Science, 2019, 494.

48. Xue Q, Tao Y, Liu Z, et al. Mechanism of oil molecules transportation in nano-sized shale channel: MD simulation[J]. RSC Advances, 2015, 5(33): 25684-25692.

49. Haixiang $\mathrm{Hu}$, Lei Du, Yanfei Xing, Xiaochun Li. Detailed study on self- and multicomponent diffusion of $\mathrm{CO} 2-\mathrm{CH} 4$ gas mixture in coal by molecular simulation[J]. Fuel, 2017, 187.

50. Chen M, Lu X, Liu X, et al. Molecular Dynamics Simulation of the Effects of $\mathrm{NaCl}$ on Electrostatic Properties of Newton Black Films[J]. Journal of Physical Chemistry C, 2012, 116(41): 21913-21922.

51. Wu G, Zhu Q, Yuan C, et al. Molecular dynamics simulation of the influence of polyacrylamide on the stability of sodium dodecyl sulfate foam[J]. Chemical Engineering Science, 2017: 313-319.

52. WU Gang, JI Xian-jing, ZHANG Tian-tian,et al. Molecular Simulation Studies on the Interaction of Polymer and Surfactant:A Review [J]. Polymer Bulletin, 2016(08): 51-60.

53. Cao Xulong. Mesoscopic simulation and design on 
dilute surfactant-polymer system[J]. Acta Petrolei Sinica(Petroleum Processing Section), 2008, 24(06): 682-688.

54. Ren Qiang, Wang Zhenyu, Sun Yuhai. Mechanism of binary complex surfactant in oil-water interface[J]. Petroleum Processing and Petrochemicals, 2018, 49(09): 43-48.

55. Li Y, Guo Y, Xu G, et al. Dissipative particle dynamics simulation on the properties of the oil/water/surfactant system in the absence and presence of polymer[J]. Molecular Simulation, 2013, 39(4): 299-308.

56. Mahdavi E, Khaledialidusti R, Barnoush A, et al. Rheological properties of super critical $\mathrm{CO} 2$ with A12O3: Material type, size and temperature effect[J]. Journal of Molecular Liquids, 2019.

57. Soleimani H, Baig M K, Yahya N, et al. Synthesis of $\mathrm{ZnO}$ nanoparticles for oil-water interfacial tension reduction in enhanced oil recovery[J]. Applied Physics A, 2018, 124(2).

58. Shaoxiang Liang, Timing Fang, Wei Xiong, et al. Oil detachment by modified nanoparticles: A molecular dynamics simulation study[J]. Computational Materials Science, 2019, 170.

59. Yan Y, Li C, Dong Z, et al. Enhanced oil recovery mechanism of $\mathrm{CO} 2$ water-alternating-gas injection in silica nanochannel [J]. Fuel, 2017: 253-259.

60. Yang Jie, Dong Zhaoxia, Xiang Qigui, et al. The pH increase mechanism of wettability alteration on sandstone surface by low saline water[J]. Journal of Northeast Petroleum University, 2018, 42(06): 104-113+11-12.

61. Sedghi Mohammad,Piri Mohammad, Goual Lamia. Atomistic Molecular Dynamics Simulations of Crude Oil/Brine Displacement in Calcite Mesopores[J]. Langmuir: the ACS

62. Jin Zhao, Guice Yao, Srinivasa B. Ramisetti, et al. Molecular dynamics investigation of substrate wettability alteration and oil transport in a calcite nanopore[J]. Fuel, 2019, 239.

63. Larson, R. G., Scriven, L. E., Davis, H. T. Monte Carlo simulation of model amphiphile-oil-water systems[J]. Journal of Chemical Physics, 1985, 83(5): 2411-2420.

64. Gunn J R, Dawson K A. A lattice model description of amphiphilic mixtures. I. Equilibrium properties[J]. Journal of Chemical Physics, 1992, 96(4): 3152-3169.

65. Wu Chuan, Zhang Rusheng, Zhang Zuguo,et al. Molecular simulation and mechanism for upgrading and viscosity reduction of extra-heavy oil[J]. Acta Petrolei Sinica, 2015, 36(03): 355-360. 